\title{
Giant intraparenchymal Neurocysticercosis: Report of surgical aspects two cases
}

\author{
Alok Umredkar, Navneet Singla, Sandeep Mohindra, Aman Bal ${ }^{1}$, Sunil Kumar Gupta \\ Departments of Neurosurgery, and ${ }^{1}$ Pathology, Postgraduate Institute of Medical Education and Research, Chandigarh, India
}

\author{
Address for correspondence: \\ Dr. Alok Umredkar, \\ Department of Neurosurgery, \\ Postgraduate Institute of \\ Medical Education and \\ Research, Chandigarh, India. \\ E-mail: alokumr@yahoo.co.in
}

DOI: $10.4103 / 0028-3886.59483$

\begin{abstract}
Giant parenchymal cysticercosis is a relatively rare condition and surgical treatment may be required when it is associated with elevated intracranial pressure. We report two patients with giant parenchymal cysticercosis who were treated surgically for the elevated intracranial pressure. In both the patients the preoperative diagnosis was of a cystic glioma. Total excision of the lesions was achieved in both the patients. In countries endemic to neurocysticercosis gaint parenchymal cysticercosis should be considered in the differential diagnosis of cystic enhancing mass lesion. Surgical excision may be indicated when it is associated with elevated intracranial pressure.
\end{abstract}

Key words: Cysticercosis, neurocysticercosis, taenia solium

\section{Introduction}

Neurocysticercosis (NCC) is the common parasitic infection worldwide. However, giant parenchymal cysticercosis, a lesion measuring more than 4 to $5 \mathrm{~cm}$ in its largest diameter and $60 \mathrm{~mL}$ in volume is uncommon. ${ }^{[1,2]}$ Giant cysts may not always be associated with elevated intracranial pressure, probably due to relatively slow growth of the lesion. ${ }^{[2]}$ Elevated intracranial pressure is an ominous sign and may require surgery. ${ }^{[2]}$ Diagnosis of NCC is often based on a combination of clinical features, radiological findings, serological and cerebrospinal (CSF) findings. ${ }^{[3]}$ The treatment of NCC is mainly medical and surgery may be indicated in patients with mass effect and elevated intracranial pressure. We report two patients with gaint intraparenchymal cysticercosis who presented to the emergency department (ED) with elevated features of elevated intracranial pressure and were treated surgically with total excision of the lesion.

\section{Case Reports}

\section{Case 1}

A 17-year girl presented to the ED with 4 months history of headache, righted sided and altered mental status of one week duration. On examination, she was drowsy and had right-sided hemiparesis (motor power grade $4 / 5$ ). Contrast magnetic resonance imaging (MRI) showed a large $(5 \times 4 \times 3.5 \mathrm{~cm})$ enhancing cystic lesion in the left insular and ganglionic region with an enhancing nodule compressing ipsilateral ventricle and perilesional edema [Figure 1a and b]. The cyst was hypointense on T1-weighted image and hyperintense on T2-weighted image. The signal intensity of cyst was similar to CSF in all the sequences. With a preoperative diagnosis of either an abscess or a cystic glioma, she was taken up for surgery. A left temporoparietal craniotomy was done and a middle temporal gyrus approach was used. At operation the lesion was firm, fibrous, avascular and cystic with clear fluid. With microsurgical technique, the cystic lesion was dissected all around and excised.
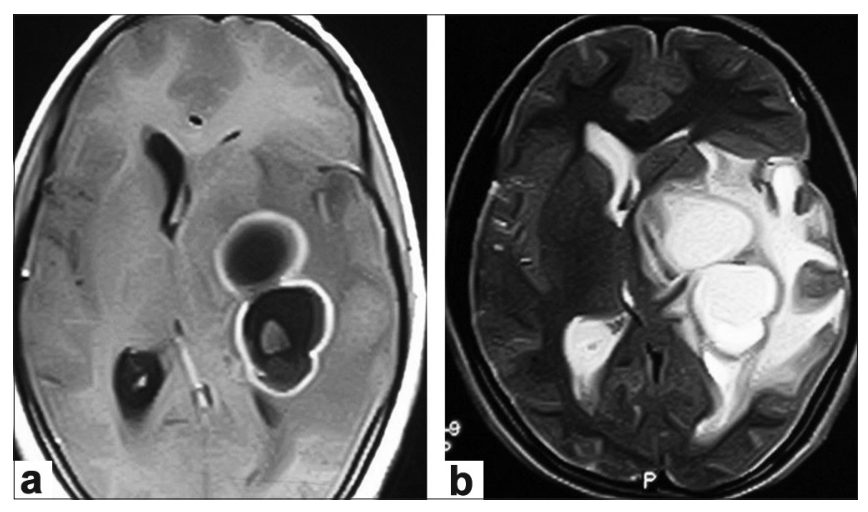

Figure 1: (a) Contrast magnetic resonance imaging scan showing ring-enhancing cystic lesion (hypointense) with enhancing nodule (scolex) in case 1; (b) T2-weighted magnetic resonance imaging scan showing same lesion (hyperintense) with marked perilesional edema in case 1 
During dissection, the cyst ruptured. Histopathology showed brain parenchyma in multiple fragments, with extensive areas of central necrosis and cysticercosus parasite. The surrounding brain parenchyma showed a dense lymphoplasmacytic infiltrate, admixed with histiocytes and eosinophils, confirming the diagnosis of cysticercal cyst [Figure 2a and b]. Postoperatively, she had worsening of motor power on the right side (grade 3/5) and transcortical motor aphasia which slowly recovered with speech therapy, physiotherapy. She was put on steroids and albendazole ( $15 \mathrm{mg} / \mathrm{kg}$ for 3 weeks). Postoperative CT scan showed total excision of the cyst [Figure 3]. At 6 months follow-up, she had recovered almost completely.

\section{Case 2}

A 40-year male presented to the ED with 2 months history of headache, seizure and altered mental status for last 1 week. On examination, he was drowsy and had right-sided hemiparesis (motor power grade 4/5). Non-contrast computerized tomography (CT) scan showed a large $(5 \times 4.5 \times 4 \mathrm{~cm})$ irregular hypodense cystic lesion in the left frontal region compressing ipsilateral ventricle and perilesional edema [Figure 4a].
Contrast MRI demonstrated a ring enhancing cystic lesion and perilesional edema and significant mass effect [Figure 4b]. As there was radiological evidence of herniation, he was taken up for surgery. The preoperative diagnostic considerations included glioma or infective lesion. At surgery, a firm, greyish, avascular cystic lesion with clear fluid was found. The lesion was excised totally. Histopathology confirmed the diagnosis of cysticercal cyst. Postoperatively, he had grade $3 / 5$ hemiparesis. Patient was put on steroids and albendazole $(15 \mathrm{mg} / \mathrm{kg}$ for 3 weeks) in the postoperative period. At 6 months follow-up, the patient had recovered fully and had no neurological deficits.

\section{Discussion}

Giant parenchymal cysticercosis is a relatively rare presentation of NCC and only few case reports have been published. ${ }^{[1-6]}$ Clinical presentation of NCC is quite variable and depends on: (i) location, growth, size and number of cysts; (ii) the stage of cyst - vesicular, degenerative, calcific; and (iii) host immune response. ${ }^{[4]}$ Seizures are the most common presentation of NCC. Diagnosis of NCC is mostly based on the clinical,
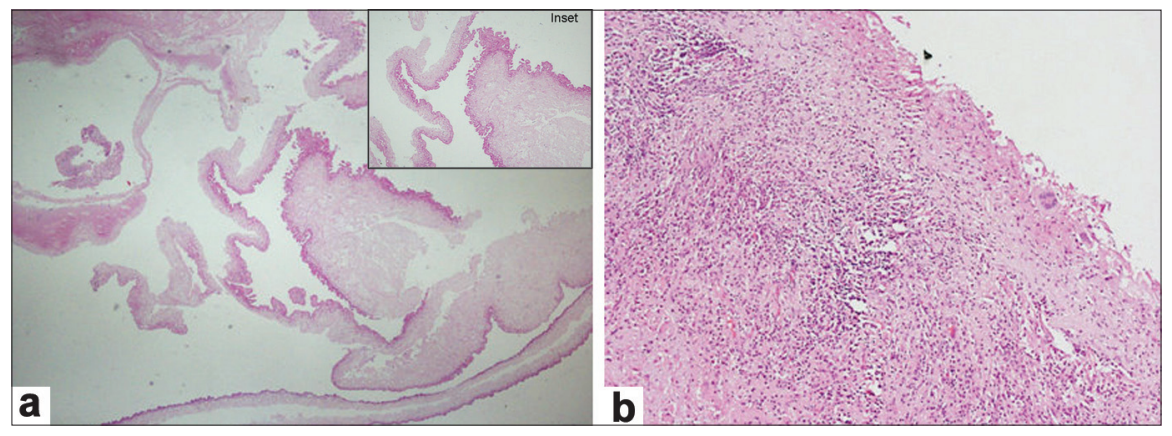

Figure 2: (a) Photomicrograph showing bladder wall of cysticercosus (H and E, 340) of case 1. Inset; showing tegumental surface having protuberances and loose connective tissue and small pyknotic nuclei beneath it $(\mathrm{H}$ and E, 3400) of case 1. (b) Photomicrograph showing palisading histiocytes, foreign body giant cells and dense inflammatory infiltrate in the surrounding brain parenchyma ( $H$ and $E, 3200)$ in case 1

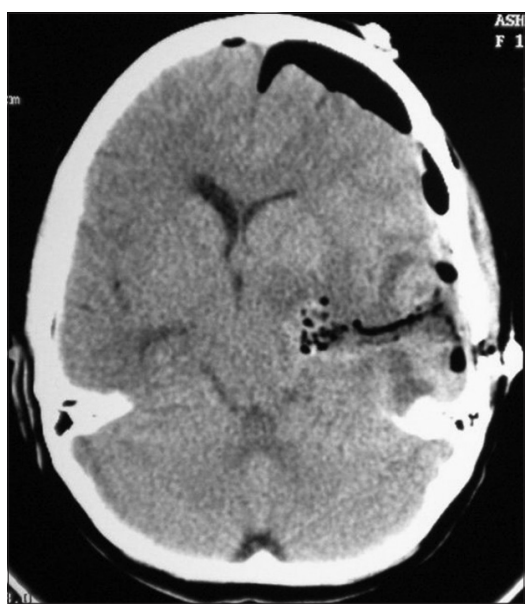

Figure 3: Postoperative NCCT showing excised cyst with postop changes in case 1
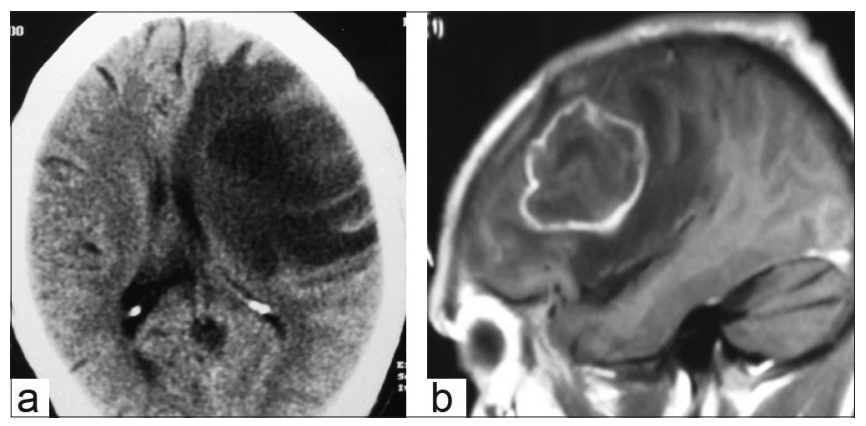

Figure 4: (a) NCCT scan showing same hypodense cystic neurocysticercosis with marked perilesional oedema with midline shift in case 2; (b) Contrast magnetic resonance imaging scan showing ringenhancing cyst (hypointense) with perilesional edema in case 2 
radiological, immunological and epidemiological parameters. ${ }^{[7]}$ The diagnostic serological test of choice in NCC is electro-immuno transfer blot assay (EITB). It has near $100 \%$ specificity and $94-98 \%$ sensitivity in patients with two or more cysts. ${ }^{[8]}$ MRI is the radiological investigation of choice to determine the pathological stage of cyst. ${ }^{[4]}$ The stage of the cyst determines the MRI cyst morphology: Viable cysts show neither enhancement nor perilesional edema, and are hypointense to brain parenchyma. Contrast enhancement and perilesional edema are the MRI characteristics of cysts in the degenerative phase. The larval cyst is recognizable as a cystic structure but is obviously irreversibly damaged, and cystic fluid becomes more turbid, corresponding to the colloidal vesicular stage described by Escobar (1983). ${ }^{[1,4]}$ Based on the radiological features there appears two types giant intraparenchymal cysticercosis: (1) non- or mildly enhancing cystic type and (2) enhancing irregular type mimicking a glioblastoma multiforme ${ }^{[7-9]}$ In the first patient the lesion was cystic type and in the second patient the lesion resembled a malignant tumor. Large cystic lesions in NCC causing elevated intracranial pressure are more commonly seen in subarachnoid spacee. Because of the rarity the diagnosis of gaint intraparenchymal cysticercosis was not considered in both the patients. However, retrospective review of the films suggested the possibility of gaint intraparenchymal cysticercosis in the first patient. There was an enhancing nodule within the cyst, probably contrast enhancing scolex. However, in the second patient there were no such clues.for diagnosing gaint cysticercosis.

The surgical approach for a large intraparenchymal cysticercosis should be by microsurgical dissection, carefully separating the thin-walled cyst from the surrounding brain parenchyma by peeling of arachnoid and saline irrigation..$^{[9]}$ No deleterious consequences secondary to intraoperative rupture were found in a study of 114 cases. ${ }^{[9]}$ In the first patient although the cyst was opened at surgery, there was no parenchymal spread of the cyst fluid. This emphasizes the need to consider gaint intraparenchymal cysticercosis in the differential diagnosis of cystic enhancing lesions. This diagnosis should be considered in endemic countries particularly in patients presenting with a large cyst with CSF-like fluid and an enhancing wall with an eccentric nodule.

\section{References}

1. Agarwal PP, Gaikwad SB, Garg A, Gupta V, Mishra NK, Mehta VS. Giant intraparenchymal neurocysticercosis: Unusual MRI findings. Neurol India 2004;52:259-60.

2. Proaño JV, Madrazo I, Avelar F, López-Félix B, Díaz G, Grijalva I. Medical treatment for neurocysticercosis characterized by giant subarachnoid cysts. N Engl J Med 2001;345:879-85.

3. Ramesh VG, Parthiban A. Giant parenchymal cysticercosis with unusual imaging features. J Clin Neurosci 2008;15:1404-6.

4. Nash TE. Human case management and treatment of cysticercosis. Acta Trop 2003;87:61-9.

5. Colli BO, Martelli N, Assirati JA Jr, Machado HR, de Vergueiro Forjaz S. Results of surgical treatment of neurocysticercosis in 69 cases. J Neurosurg 1986;65:309-15.

6. Soto Hernández JL, Ostrosky Zeichner L, Tavera G, Gómez Aviña A. Neurocysticercosis and HIV infection: Report of two cases and review. Surg Neurol 1996;45:57-61.

7. Del Brutto OH, Rajshekhar V, White AC Jr, Tsang VC, Nash TE, Takayanagui OM, et al. Proposed diagnostic criteria for neurocysticercosis. Neurology 2001;57:177-83.

8. Garg RK. Diagnostic criteria for neurocysticercosis; some modifications are needed for Indian patients. Neurol India 2004;52:171-7.

9. Escobedo F. Neurosurgical aspect of neurocysticercosis. In Schmidek H, Sweet W, editors. Operative neurosurgical techniques. $4^{\text {th }} \mathrm{ed}$. Philadelphia: WB Saunders; 2000. p. 1756-68.

Accepted on 09-10-2009

Source of Support: Nil, Conflict of Interest: None declared. 